CONCISE REPORT

\title{
Effect of the first infliximab infusion on sleep and alertness in patients with active rheumatoid arthritis
}

\author{
C Zamarrón, F Maceiras, A Mera, J J Gómez-Reino
}

Ann Rheum Dis 2004;63:88-90. doi: 10.1136/ard.2003.007831

\begin{abstract}
Objective: To assess the benefit of the first infliximab infusion on sleep disturbances in patients with RA

Material and methods: Evaluation of RA activity, sleepiness (Epworth scale and multiple sleep latency test), alertness (steer clear test), and sleep structure (polysomnography) were conducted before and after the first infusion of infliximab in six female patients with RA.

Results: The day after the first infliximab infusion, the mean (SD) number of tender (20 (2.4)) or swollen (15.3 (2)) joints and the morning stiffness (140 (61.9) min) had not changed. There were significant improvements in the median number of total sleep stage transitions per hour (median (IR) before $v$ after infusion: 20.5 (43) v 7.5 (6); Wilcoxon paired test, $\mathrm{p}=0.014)$, median percentage of phase $\mathrm{I}+\mathrm{II}(83.5(8) \vee 54.5$ (24); $p=0.023)$, percentage of REM stages (2 (10) $v 11.5$ (8); $p=0.014)$, median percentage sleep efficiency (44 (22) v 75 (18); $p=0.014)$, median sleep latency (77.5 (150) $v$ 25.5 (23) min; $p=0.023)$, and median number of hits in the steer clear test $(48.5(86) \vee 6(45) ; p=0.023)$. Neither objective nor subjective daytime sleepiness was noted. One obese patient had obstructive sleep apnoea syndrome.

Conclusions: Sleep and the alertness disturbances in RA improve with infliximab treatment. Improvement appears unrelated to joint discomfort amelioration but suggests a central effect through inhibition of circulating TNF $\alpha$ levels.
\end{abstract}

$\mathrm{T}$ umour necrosis factor $\alpha(\mathrm{TNF} \alpha)$ mRNA and receptors for $\mathrm{TNF} \alpha$ are expressed in normal as well as diseased brain tissue. ${ }^{1}$ In humans and animal models, TNF $\alpha$ is involved in normal and disturbed sleep. Circulating and locally produced $\mathrm{TNF} \alpha$, when abnormal, disrupts sleep patterns. ${ }^{2}$

Studies in rheumatoid arthritis (RA) have documented disruption in sleep continuity as well as qualitative alterations in the electroencephalographic (EEG) activity. ${ }^{3}$ There is a relationship between changes in the structure of sleep and certain immunological factors. In RA, circulating levels of $\mathrm{TNF} \alpha$ are increased, ${ }^{4}$ and there has been speculation that the circulating level of TNF $\alpha$ is one of the elements causing the sleep disorders that have been noted in patients with this condition. This study was conducted to assess whether infliximab, a chimeric monoclonal antibody to TNF $\alpha$ with proven efficacy in the treatment of RA, ${ }^{5}$ has an effect on the disturbances of sleep patterns seen in patients with RA.

\section{PATIENTS AND METHODS}

\section{Patients}

Six female patients with RA, defined according to the American College of Rheumatology 1987 revised criteria, resistant to treatment with methotrexate, who had a large number of inflamed and painful joints, and who were scheduled for treatment with infliximab, were asked to participate in this non-controlled study. The patients had no history of tobacco use or any metabolic or neurological abnormality. Table 1 presents further clinical and demographic data. All participants provided written informed consent to participation in the study.

\section{Study design}

In this non-controlled prospective study, measurements were conducted during two days of hospitalisation, before (baseline) and after infliximab infusion. In two patients the study included an overnight stay on the ward before the infliximab infusion. Infliximab at a dose of $3 \mathrm{mg} / \mathrm{kg}$ body weight was given intravenously over two hours after the initial baseline clinical measurements.

Evaluation of RA activity was on the evening before and the morning after the infliximab administration. Over the course of the morning, the degree of body stiffness was described by the patient, the number of tender or painful joints (possible total 68), the number of swollen joints (possible total 66), and fatigue measured by a visual analogue scale were recorded.

The degree of sleepiness was subjectively measured with the Epworth sleepiness scale. ${ }^{6}$ The level of vigilance while driving and the ability of the subject to perform a task requiring protracted alertness was investigated using a computer program designed for the task. ${ }^{7}$ All the patients were tested before the sleep study as well as before and after the infliximab infusion between 1500 and 1800 hours. Performance was categorised in three groups according to the percentage of obstacles hit: normal, $<1.8 \%$ of obstacles hit; poor, between $1.8 \%$ and $4.5 \%$ of obstacles hit; and very poor performance, $>4.5 \%$ of obstacles hit.

Sleep studies were carried out in the sleep unit. An arousal was defined according to the American Sleep Disorders Association. ${ }^{8}$ A standard multiple sleep latency test was carried out and consisted of a series of $4 \times 20$ minute nap trials at two hourly intervals.

\section{Statistical analyses}

For descriptive statistics, the median values with interquartile ranges (IR) were used and the non-parametric Wilcoxon test for paired samples was used for comparisons.

\section{RESULTS}

Table 1 summarises the characteristics of the patients. Comparison of these clinical measures before and after infliximab treatment showed no statistically significant changes

The most significant sleep abnormality, in all patients, was fragmentation. They had high sleep latency, low sleep

Abbreviations: EEG, electroencephalography; OSA, obstructive sleep apnoea; RA, rheumatoid arthritis; TNF $\alpha$, tumour necrosis factor $\alpha$ 
Table 1 Clinical characteristics of six patients with RA before and after treatment with infliximab

\begin{tabular}{|c|c|c|c|c|c|c|c|c|}
\hline \multirow[b]{2}{*}{ Patient } & \multirow[b]{2}{*}{ Age (years) } & \multicolumn{2}{|c|}{ Tender joints (n) } & \multicolumn{2}{|c|}{ Swollen joints (n) } & \multicolumn{2}{|c|}{ Morning stiffness (min) } & \multirow[b]{2}{*}{ ESR $(\mathrm{mm} / 1 \mathrm{st} h)$} \\
\hline & & Day 1 & Day 2 & Day 1 & Day 2 & Day 1 & Day 2 & \\
\hline 1 & 65 & 21 & 20 & 19 & 17 & 60 & 45 & 30 \\
\hline 2 & 42 & 19 & 21 & 15 & 11 & 120 & 90 & 55 \\
\hline 3 & 32 & 16 & 16 & 14 & 13 & 120 & 120 & 34 \\
\hline 4 & 62 & 24 & 21 & 16 & 16 & 180 & 180 & 37 \\
\hline 5 & 52 & 23 & 22 & 13 & 14 & 240 & 240 & 43 \\
\hline 6 & 71 & 20 & 20 & 14 & 13 & 180 & 180 & 36 \\
\hline Median (IR) & 57 (17) & $20.5(4)$ & $20.5(1)$ & $15(2)$ & $13.5(4)$ & $150(60)$ & $150(90)$ & 38.5 (9) \\
\hline
\end{tabular}

ESR, erythrocyte sedimentation rate.

Day 1 = morning before infliximab infusion; day $2=$ morning after infliximab infusion.

efficiency, high percentages of phase I+II stages, reduced deep sleep stages, and an abnormally high number of arousals (tables 2 and 3), indicating a fragmented and poor quality sleep. None of the patients showed pathological daytime sleepiness (table 3). Poor performance in the steer clear driving test was seen in five patients. Two patients had the above studies done two nights before the infliximab infusion and no statistically significant differences were observed in the recordings. Polysomnography indicated that one obese patient had obstructive sleep apnoea (OSA) syndrome.

After infliximab infusion there was a significant improvement in the median number of total sleep stage transitions per hour (median (IR) before $v$ after infusion 20.5 (43) $v 7.5$ (6); Wilcoxon paired test, $\mathrm{p}=0.014)$, median percentage of phase I+II (83.5 (8) $v 75$ (18); $\mathrm{p}=0.023)$, percentage of REM stages $(2(10) v 11.5(8) ; \mathrm{p}=0.014)$, median percentage sleep efficiency ( $44(22) \vee 75$ (18); $\mathrm{p}=0.014)$, median sleep latency in minutes $(77.5(150) \vee 25.5(23) ; \mathrm{p}=0.023)$ and median number of hits in steer clear test $(48.5$ (86) $v 6$ (45); $\mathrm{p}=0.023)$.

In the patient with OSA sleep efficiency improved from $67 \%$ to $80 \%$, REM stages dropped from 336 minutes to 85 minutes, the number of transition sleep stages decreased from 11 to 2, and arousals fell from 14 to 4 an hour. These changes were accompanied by drop in the mean $\mathrm{O}_{2}$ saturation from $91 \%$ to $86 \%$ and an increase in the hypopnoea/apnoea index from 55 to 74 .

\section{DISCUSSION}

In this study we investigated the effect of infliximab on sleep pattern disturbances in six patients with active RA. Sleep abnormalities improved after the initial dose of the antibody. Despite the lack of controls, our data demonstrate sufficient consistency. Firstly, other studies have reported similar sleep disturbances in ambulatory and hospitalised patients with RA. ${ }^{3}$ Secondly, the "first night effect" is mild and does not involve sleep disordered breathing and the steer clear test. ${ }^{9}$
Although patients may sleep better at home, there is no evidence that it makes any difference when the patterns are recorded. For example, in two of our patients we made the recordings two nights before treatment with infliximab and the analysis indicated no relevant differences. Thirdly, improvement in our patients was uniform, rapid and consistent.

EEG and sleep disturbances are common in RA. A possible explanation is that the disease may cause both pain and sleep disturbances through the release of cytokines. In another study in patients with RA using polysomnography, ${ }^{3}$ sleep was disrupted regardless of the inflammatory activity of the disease and, interestingly, most patients had severe sleep fragmentation. The reported EEG as well as sleep disturbances match those seen in our study and, additionally, we noticed a deterioration of cognitive performance (vigilance and reaction time) as measured by the steer clear test. Previous studies have shown that impaired vigilance correlates with sleep fragmentation, which was common in our patients, but not with daytime sleepiness. ${ }^{10}$ This would explain the presence of sleep fragmentation, deterioration of vigilance, and absence of daytime sleepiness encountered in all of our patients except the one with sleep apnoea. Taken together, the information gathered indicates that deterioration of vigilance was due, at least in part, to sleep fragmentation.

After infliximab, there was an improvement in the fragmented and poor quality sleep characterised by high sleep latency, low sleep efficiency, high percentages of I+II stages, reduced percentages of deep sleep stages (phase III+IV), and an abnormally large number of arousals. This was paralleled by the amelioration of vigilance in the patients with the more anomalous steer clear test. Of note was the improvement of vigilance and sleepiness that was seen even in the patient with OSA despite the increase in the dips in oxygen saturation and apnoeas. The prompt response to the antibody suggests that TNF $\alpha$ was a key factor in the sleep disturbances. Raised circulating levels of TNF $\alpha$ are found in

Table 2 Sleep parameters of the six patients with RA before and after treatment with infliximab

\begin{tabular}{|c|c|c|c|c|c|c|c|c|c|c|c|c|}
\hline \multirow[b]{2}{*}{ Patient } & \multicolumn{2}{|c|}{ Sleep latency (min) } & \multicolumn{2}{|c|}{ REM$^{*}$ latency ( $\mathrm{min}$ ) } & \multicolumn{2}{|c|}{ Sleep efficiency (\%) } & \multicolumn{2}{|c|}{ Phase I+II (\%) } & \multicolumn{2}{|c|}{ Phase III+IV (\%) } & \multicolumn{2}{|c|}{ REM (\%) } \\
\hline & Pre & Post & Pre & Post & Pre & Post & Pre & Post & Pre & Post & Pre & Post \\
\hline 1 & 97 & 3 & 186 & 73 & 36 & 86 & 83 & 30 & 7 & 55 & 10 & 15 \\
\hline 2 & 172 & 36 & - & 134 & 39 & 70 & 94 & 37 & 6 & 52 & 0 & 11 \\
\hline 3 & 197 & 24 & - & 288 & 32 & 58 & 84 & 61 & 16 & 22 & 0 & 7 \\
\hline 4 & 58 & 38 & - & 106 & 58 & 68 & 88 & 58 & 12 & 30 & 0 & 12 \\
\hline 5 & 32 & 13 & 113 & 61 & 55 & 93 & 80 & 51 & 4 & 32 & 16 & 17 \\
\hline 6 & 24 & 27 & 336 & 85 & 67 & 80 & 80 & 88 & 16 & 6 & 4 & 6 \\
\hline Median IR) & 77.5 (150) & $25.5(23)$ & - & - & 44 (22) & 75 (18) & $83.5(8)$ & 54.5 (24) & $9.5(10)$ & 31 (30) & $2(10)$ & $11.5(8)$ \\
\hline
\end{tabular}

Pre $=$ the night before infliximab infusion; post = the night after the infliximab infusion . 
Table 3 Sleep and alertness parameters of the six patients with RA before and after treatment with infliximab

\begin{tabular}{|c|c|c|c|c|c|c|c|c|c|c|}
\hline \multirow[b]{2}{*}{ Patient } & \multicolumn{2}{|c|}{ Arousals/h } & \multicolumn{2}{|l|}{ SST/h } & \multicolumn{2}{|c|}{ Steer clear hits* } & \multicolumn{2}{|c|}{ Epworth scale* } & \multicolumn{2}{|c|}{ Fatigue scale* } \\
\hline & Pre & Post & Pre & Post & Day 1 & Day 2 & Day 1 & Day 2 & Day 1 & Day 2 \\
\hline 1 & 45 & 8 & 30 & 16 & 171 & 92 & 7 & 4 & 5.2 & 5 \\
\hline 2 & 15 & 11 & 62 & 7 & 96 & 48 & 6 & 6 & 5.9 & 6 \\
\hline 3 & 7 & 9 & 9 & 6 & 19 & 3 & 4 & 4 & 3.5 & 3 \\
\hline 4 & 9 & 11 & 54 & 12 & 37 & 2 & 3 & 4 & 7 & 6.4 \\
\hline 5 & 10 & 5 & 11 & 8 & 3 & 6 & 3 & 2 & 5.1 & 5.6 \\
\hline 6 & 14 & 4 & 11 & 2 & 60 & 6 & 14 & 4 & 5.6 & 5.5 \\
\hline Median (IR) & $12(6)$ & $8.5(6)$ & $20.5(43)$ & $7.5(6)$ & $48.5(86)$ & $6(45)$ & $5(4)$ & $4(0)$ & $5.4(0.8)$ & $5.7(0.6)$ \\
\hline
\end{tabular}

Pre = the night before infliximab infusion; post = the night after infliximab infusion; day $1=$ morning before infliximab infusion; day $2=$ morning after infliximab infusion; SSR, sleep stage transitions.

*See text for descriptions.

RA and other conditions, such as HIV-AIDS, where alterations in sleep have been documented. ${ }^{11}$ It may be argued that the benefits observed in sleep improvement result from improvement in joint inflammation and pain that the patient experiences. However, a recent report ${ }^{12}$ found no benefit in sleep even when a significant clinical improvement in arthritis was achieved after treatment with tenoxicam. Additional evidence is our observation of an improvement in EEG and steer clear test performance which occurred the night after the infliximab administration, even when there were no objective signs of joint pain amelioration. Further support comes from the observation of the positive effect of infliximab on sleep fragmentation in the patient with OSA. TNF $\alpha$ induces leptin production, ${ }^{13}$ whereas a block of TNF $\alpha$ could cause a drop in leptin concentrations. In humans and animal models, leptin deficiency appears to have an active role in OSA, ${ }^{14}$ but the levels of this oroxigenic peptide were not measured in our patient. Reduced upper airway muscle tone is a major cause of OSA. Other common causes in RA, such as brain stem compression, upper airway obstruction from micrognathia, and the abuse of alcohol and sedatives or hypnotic drugs, were not present in our patient. Increasing respiratory effort is known to induce repeated arousals, and the number of arousals decreased in all our patients after infliximab infusion, including the patient with OSA. As a result, in this patient there was increased apnoea and recurrent oxyhaemoglobin desaturation. Hence, in patients with RA who are to be treated with infliximab, a careful history of sleep alterations needs to be documented so as to identify those with OSA. Sleep is also regulated by melatonin and the nocturnal levels of this peptide are increased in RA. ${ }^{15}$ Whether the improvement of sleep parameters relates to the modulation of melatonin through $\mathrm{TNF} \alpha$ is unknown at present.

In summary, the results of this study suggest that the sleep and alertness disturbances in patients with RA improve with the use of infliximab. In patients with RA who have OSA oxygen desaturation may worsen despite this improvement in sleep structure. The improvement does not appear to reflect joint pain amelioration but seems rather to be a central nervous system effect due to inhibition of raised circulating levels of TNF $\alpha$. It should be noted that the results were obtained in a group of patients with well documented RA with persistent activity despite treatment with a disease modifying drug and that these preliminary conclusions pertain exclusively to this selected patient group. Our results need further confirmation in a more extensive controlled study.

\section{Authors' affiliations}

C Zamarrón, The Sleep Unit of the Respiratory Service, Hospital Clinico Universitario and Medical School, Universidad de Santiago de Compostela, Spain

F Maceiras, A Mera, J J Gómez-Reino, Rheumatolology Service, Hospital Clinico Universitario and Medical School, Universidad de Santiago de Compostela, Spain

A Mera, J J Gómez-Reino, Department of Medicine, Hospital Clinico Universitario and Medical School, Universidad de Santiago de Compostela, Spain

Correspondence to: Professor J J Gómez-Reino, Rheumatology Service, Hospital Clínico Universitario, C/Choupana s/n, 15706 Santiago, Spain; juan.gomez-reino.carnota@sergas.es

Accepted 12 May 2003

\section{REFERENCES}

1 Szelenyi J. Cytokines and the central nervous system. Brain Res Bull 2001;54:329-38

2 Krueger JM, Obal FJ, Faig J, Kubota T, Taishi P. The role of cytokines in physiological sleep regulation. Ann N Y Acad Sci 2001;933:211-21.

3 Hirsch M, Carlander B, Verge M, Tafti M, Anaya JM, Billiard M, et al. Objective and subjective sleep disturbances in patients with RA: a reappraisal. Arthritis Rheum 1994;37:41-9.

4 Feldman M, Brennan FM, Maini RN. Role of cytokines in RA. Annu Rev Inmunol 1996; 14:397-440.

5 Maini RN, Breedveld FC, Kalden JR, Smolen JS, Davis D, Macfarlane JD, et al. Therapeutic efficacy of multiple intravenous infusions of anti-tumor necrosis factor a monoclonal antibody combined with low-dose weekly methotrexate in RA. Arthritis Rheum 1998;41:1552-63.

6 Johns MW. A new method for measuring daytime sleepiness: the Epworth sleepiness scale. Sleep 1991;14:540-5.

7 Findley LJ, Fabrizio MJ, Knight H, Norcross BB, Laforte AJ, Suratt PM. Driving simulator performance in patients with sleep apnea. Am Rev Respir Dis 1989; 140:529-30.

8 American Sleep Disorders Association. The atlas task force. EEG arousals: scoring rules and examples. Sleep 1992;9:519-24.

9 Mosko SS, Dickel MJ, Ashurst J. Night to night variability in sleep apnea and sleep-related periodic movements in the elderly. Sleep 1988;11:340-8.

10 Engleman H, Martin S, Deary I, Douglas NJ. Effect of continuous positive airway pressure treatment on daytime function in sleep apnea/hypopnea syndrome. Lancet 1994;342:572-5.

11 Darko DF, Miller JC, Gallen C, White J, Koziol J, Brown SJ, et al. Sleep electroencephalogram delta frequency amplitude, night plasma levels of TNFalpha, and human immunodeficiency virus infection. Proc Natl Acad Sci USA 1995;92:12080-4.

12 Lavie P, Nahir M, Lorber M, Scharf Y. Non-steroidal anti-inflammatory drug therapy in RA patients. Lack of association between clinical improvement and effects on sleep. Arthritis Rheum 1991;34:655-9.

13 Polotsky VY, Wilson JA, Smaldone MC, Haines AS, Hurn PD, Tankersley CG, et al. Tumor necrosis factor (TNF)-alpha induces leptin production through the p55 TNF receptor. Am J Physiol Regul Integr Comp Physiol 2000;278:R537-43.

14 Finck BN, Johnson RW. Female gender exacerbates respiratory depression in leptin-deficient obesity. Am J Respir Crit Care Med 2001;164:1470-5.

15 Sulli A, Maestroni GJ, Villaggio B, Hertens E, Craviotto C, Pizzorni C, et al. Melatonin serum levels in rheumatoid arthritis. Ann NY Acad Sci 2002;966:276-83. 